\title{
La conservación y restauración del patrimonio Entre la orfandad formativa y la falta de definición facultativa
}

\author{
Javier Bueno Vargas \\ Conservador-restaurador \\ Vicedecano del Colegio Oficial de Doctores y Licenciados en \\ Bellas Artes de Andalucía
}

Podemos afirmar que además de ser una asignatura pendiente la formación y el desarrollo profesional de los conservadores en este País, es hasta este momento asignatura suspensa. Efectivamente, la remodelación de los planes de estudios universitarios de la carrera desde la que se formaban los restauradores, la Licenciatura en Bellas Artes y la consecuente desaparición de sus especialidades, ha reducido la carga lectiva dedicada a la formación de conservadores-restauradores en estos estudios superiores, desapareciendo así esta especialidad en favor de un título general de "Licenciado en Bellas Artes". Esto está obligando a reagrupar o suprimir parte de la carga docente, incluida gran parte de las asignaturas de restauración; como consecuencia de lo anterior, se impide una profundización en este tipo de formación con el nivel de especialización con el que antes se hacía. Sólo la libre elección curricular de los estudiantes entre las asignaturas optativas ofertadas posibilita cierta especialización en conservación y restauración siempre dentro de esta titulación general. Además nos encontramos con la proliferación de academias, escuelas taller, centros privados y religiosos o estudios de postgrado de experto y masters de todo tipo en donde se imparten enseñanzas de conservación y restauración a distintos niveles y a todo tipo de público, lo que evidentemente redunda en detrimento de los profesionales especializados favoreciendo el intrusismo y, generalmente, la inadecuada manipulación y tratamiento del Patrimonio.

Y, ¿qué está pasando en la Administración?, pues como veremos, la profesionalización y acceso al funcionariado, incluida la creación del cuerpo técnico superior de conservadores-restauradores, siguen siendo materias pendientes desde hace ya dos décadas.

Es indiscutible que la conservación y restauración del Patrimonio Cultural es una actividad y preocupación fundamental en toda sociedad contemporánea, tanto como elemento clave en la identidad colectiva, como motor de desarrollo. Así desde la formulación de la Constitución Española, vigente desde 1978, se reconoce que el acceso a la cultura es un derecho que los poderes públicos promoverán y tutelarán I. Además recoge, en su artículo 46, que "Los poderes públicos garantizarán la conservación y promoverán el enriquecimiento del patrimonio histórico, cultural y artístico de los pueblos de España y de los bienes que lo integran". Se convierte así nuestro Patrimonio en un derecho de los ciudadanos debiendo la administra- ción facilitar los cauces y a los profesionales que velen por esta salvaguardia. La Ley del Patrimonio Histórico Español 16/1985 ratifica este planteamiento profundizando en la normalización.

Por su parte, la Administración de la Comunidad Autónoma Andaluza asumió con su Estatuto de Autonomía de 1981 y a través del Real Decreto 864/1984 las competencias exclusivas referidas al Patrimonio Cultural, con lo que se responsabilizaba así de todo lo concerniente a la protección y conservación del Patrimonio. Para cumplir con sus nuevas obligaciones en el caso del tratamiento de bienes muebles, la Consejería de Cultura incluso debió crear un Departamento de Bienes Muebles integrado en el Servicio de Conservación y Restauración ya que este servicio no existía con anterioridad.

Es evidente que los niveles de concienciación, de relación, de preocupación e interés social por el Patrimonio ya no son el que nos podíamos encontrar hace dos décadas. Así el gobierno autonómico ha logrado implicar a la sociedad de diversas formas entre ellas promoviendo y difundiendo su preocupación e intenciones mediante la generación documentos como las "Bases para una Carta sobre Patrimonio y Desarrollo en Andalucía"2, con el que se pretendía no sólo apuntar "el camino que deben seguir las políticas sobre Patrimonio en el futuro"(pág. 5), sino sobre todo intentar trasladar el debate del Patrimonio como factor de desarrollo al conjunto de la sociedad andaluza, por cuanto que ese Patrimonio no existiría como tal si "no es asumido por un colectivo que se reconoce en él, y no habrá política patrimonial correcta si este colectivo no adopta una actitud participativa, crítica, constructiva y creativa"(pág. 5). En esta estrategia ha sido fundamental la formulación de los dos Planes Generales de Bienes Culturales como instrumentos de actualización en la planificación de la tutela del Patrimonio Andaluz: el primero para el período 1989-1995 y el segundo para el período 1996-2000. Ambos se proponían como "instrumento político para la actuación en ese campo complejo del Patrimonio y comprende tanto el aparato conceptual como la preparación de una estructura administrativa que permita llevarlo a cabo" 3 .

Pero pasaron el primer y segundo Plan General de Bienes Culturales, de cuyos planteamientos y propuestas esperamos la correspondiente evaluación conjunta y, si los analizamos detenidamente centrándonos en las propuestas sobre la formación y regulación profesional de los conservadores-restauradores, entenderemos la necesidad de formular el tercero cuya existencia ya se recogía desde el Parlamento de Andalucía 4 para el período 200 I-2004, con una nueva programación de objetivos y líneas de actuación. 


\section{El primer plan general de Bienes Culturales (1989-1995) y la Formación y Profesionaliza- ción de los Conservadores-Restauradores}

El primer Plan andaluz consistía en un texto de poco más de 100 páginas, complementadas con el listado de Bienes Inmuebles y Muebles declarados o incoados como BIC. Si lo repasamos nos parece observar que para la Administración la principal preocupación en ese momento era sobre todo cuantificar los Bienes de los que se había responsabilizado cinco años antes. En referencia al tema de la formación y profesionalización de los restauradores apunta como se estaban perdiendo conocimientos en los oficios que intervienen en la conservación y restauración y parecía no existir un número adecuado de profesionales de alto nivel de especialización en el campo de la tutela, conservación o restauración de los Bienes Culturales (pág. 18).

Efectivamente, en nuestra Comunidad se estaban perdiendo numerosos oficios artesanales, ya fuera por la pérdida de estatus de este sector como por el aparente desinterés en ese momento de la Administración por recuperar este tipo de formación y dotarla del reconocimiento social que merecía. Sin embargo la formación oficial de restauradores, a través de las facultades de Bellas Artes y por tanto con rango de licenciatura, se venía impartiendo en Andalucía desde 198I en la Universidad de Sevilla (y por tanto ya había cinco promociones insertadas en el mundo laboral), centro al que se unió después la Facultad de Bellas Artes de Granada, que había iniciado su andadura en 1986 y por tanto estaba insertando profesionalmente a restauradores desde 1990. Además recordemos que estas enseñanzas se impartían ya desde 1942 cuando se organiza la Escuela Superior de Bellas Artes que, a través de su artículo 7, creaba la sección de Restauración; con rango universitario ya se formaban también restauradores desde otros centros universitarios de Barcelona, Bilbao, Madrid (también desde 1981) y Valencia (desde 1989). No nos olvidemos que existía ya la Escuela Superior de Conservación y Restauración de Madrid (desde 1987), estudios que alcanzan en este caso el rango de diplomatura .

No se entiende por tanto este comentario en referencia a la falta de profesionales cualificados cuando en general los planes de estudios desarrollados contemplaban una especialización mediante tres años de estudios generales en Bellas Artes y dos específicos en Conservación y Restauración, (que es de tres años en el caso de las escuelas mencionadas), además de contar con programas de investigación a través del tercer ciclo y doctorado. Quizá se pensara que no existía suficiente especialización porque en las directrices generales de estos estudios de Licenciatura en Bellas Artes6, no se detallaron los requisitos mínimos del currículo de Conservación-Restauración de Bienes Culturales, lo que no se hace en el ámbito nacional hasta 1991, (en el caso de los estudios a desarrollar desde las Escuelas de Restauración7); en el caso de los estudios universitarios no es hasta 1994 cuando el Consejo de Univer- sidades reconoce la necesidad de incorporar el título específico de Licenciado en Conservación y Restauración, aunque al día de hoy no se hayan creado dichos estudios. Sin embargo, los restauradores licenciados en Bellas Artes sí estaban preparados si repasamos los planes de estudios que se desarrollaban, otro caso es la desastrosa hacia la que se dirige la formación ahora y que veremos más adelante.

En lo referente a formación y profesionalización de la restauración no se menciona nada en este Plan. El capítulo 5, sobre el programa de conservación y restauración de bienes culturales se centra, en el área de programación ordinaria, en la problemática del tratamiento de inmuebles y de los responsables de estas intervenciones. De entre los profesionales que intervienen (pág. 67) sólo se hace mención a los arquitectos y al grupo de técnicos que se van a encargar del edificio (y que realizarán una ficha diagnóstico complementaria). Sin embargo, entre los objetivos de este área se vuelve a hacer mención a la necesidad de desarrollar el área de proyectos de intervención mediante la "formación de especialistas en el campo de la restauración y conservación de bienes muebles e inmuebles mediante becas, cursos, seminarios y congresos" (pág. 7l), o la también ya mencionada necesidad de recuperar oficios artesanales para acometer el mantenimiento necesario en el caso del patrimonio inmueble, a través de la Formación Profesional y las Escuelas-Taller. Nuevamente se obvia la preparación oficial ya existente.

En lo referente al tratamiento de Bienes Muebles (pág. 76), se menciona, a nivel profesional, "la ausencia de personal especializado en el ámbito de la Administración que permita desarrollar las funciones asignadas por la Ley de Patrimonio" y se insiste una vez más en "la falta de profesionales especializados en las distintas ramas que configuran los Bienes Muebles en materia de Conservación y Restauración". El primero de los objetivos del Plan de Conservación y Restauración de Bienes Muebles (pág. 76) volverá a ser la "formación de un personal especializado (...) que posibilite la dotación de una infraestructura que cubra progresivamente las necesidades de intervención en las diferentes áreas" y que se potenciará a través del mercado de trabajo existente (cuya cualificación profesional esté reconocida por la Administración), de cursos y seminarios, de la determinación de objetivos y técnicas en los programas ocupacionales de las Escuelas-Taller y en la Formación Profesional y en otros similares, colaborando en la preparación de los profesionales y controlando las intervenciones en este ámbito y finalmente mediante la formación de un registro de profesionales en este sector.

Es redundante el constatar la aparente falta de profesionales y se manifiesta una confusión en el grado de responsabilidad y cualificación de los distintos técnicos con los que se debe contar como ejecutores de las intervenciones de restauración, es decir, entre los técnicos superiores (licenciados) y los auxiliares (diplomados, ayudantes, artesanos). 
Si seguimos repasando este primer Plan, vemos como la Administración reconoce en el Programa de Instituciones del Patrimonio Histórico (pág. 84) que "urge, en efecto, aportar los recursos humanos y técnicos necesarios para el desarrollo de las tareas de conservación, cuya finalidad es imposible cumplir si la institución (museística) no puede llevar a cabo tareas de protección o restauración de urgencia o mantenimiento". Pero entre los objetivos a cumplir en este punto no aparece la dotación de los talleres de restauración y sí se apunta nuevamente la necesidad de la formación de nuevos profesionales de los museos (en general) a través del IAPH. En cuanto al Plan de los Conjuntos Arqueológicos y Monumentales se recoge la necesidad de dotarlos entre otras cosas, del equipamiento técnico necesario incluyéndose un apartado de restauración (pág. 87) y destacando entre las funciones del IAPH una vez más la de la formación "para crear un cuerpo de especialistas de alta cualificación profesional que garantice una tutela efectiva de los bienes culturales en el área de nuestra Comunidad"(pág. 90). Se describen también las labores del IAPH en cuanto a intervención del patrimonio, (se hace mención a los programas de trabajo a realizar), pero nada se dice de la consolidación profesional de los técnicos que las llevan a cabo ni de la posibilidad de su estabilización como personal de plantilla dentro de la Administración.

La confusión sobre las distintas cualificaciones profesionales (y los grados de formación y competencias con las que deben contar), vuelve a quedar patente al recogerse, dentro de las funciones del Patronato de la Alhambra y el Generalife de Granada8, el que entre ellas estará el "impulsar la formación de personal artesano, para atender las propias necesidades de conservación y restauración de los monumentos del recinto".

Además de esta falta de definición de las categorías profesionales resulta contradictorio que siendo la partida de conservación y restauración la que recibió una dotación económica mayor, 37.843 millones de pesetas (37,4\% del gasto global del Plan General ${ }^{9}$, de ellos sólo estuviera previsto en esos 7 años dedicar cantidades tan insuficientes como 174 millones para gastos de funcionamiento: personal, gastos corrientes, realización de contratos específicos y a la urgente formación de especialistas de la que insistentemente se hablaba y de la que se quejaba el propio Plan; o los tan sólo I5 millones destinados al capítulo de inversión nueva, partida en la que se incluía la adquisición de bienes culturales muebles e jinmuebles!, y también el equipamiento de talleres, laboratorios, etc., infraestructura técnica y administrativa que según se recogía 10, no habían sido heredadas con el traspaso de competencias de la Administración Central a la Consejería de Cultura y como hemos señalado en los párrafos anteriores, se planteaba como necesidad prioritaria. Efectivamente como reconoce en el Plan General de Bienes Culturales 1989-1995, (pág. 90), a finales de los 80, en relación con los bienes culturales, la Administración estaba infradotada de personal suficientemente cualificado y por ello se contaba "funda- mentalmente con profesionales libres" y con la colaboración de departamentos universitarios o laboratorios de empresas especializadas. Con esa dotación presupuestaria parecía evidente que en ese momento no existía interés por cambiar esta situación creando un cuerpo profesional de restauradores dentro de la Administración.

En ejecución del Plan General de Bienes Culturales para el período 1989-1995 se promulgó la Ley |/|99|, de 3 de julio, de Patrimonio Histórico de Andalucía, texto legal que abordó, de una manera global, la tutela del Patrimonio Histórico Andaluz. Esta Ley ha constituido el instrumento normativo básico para el desarrollo de la política de Bienes Culturales en la Comunidad Autónoma Andaluza, completado por la reglamentación de desarrollo, contenida en tres grandes Reglamentos dedicados respectivamente a la Organización Administrativa, a las medidas de Protección y Fomento y a las Actividades Arqueológicas, quedando aún pendiente el Reglamento de Conservación cuya consecución tantos problemas evitaría y solucionaría.

\section{El Segundo Plan General de Bienes Culturales (1995-2000) y la Formación y Profesionaliza- ción de los Conservadores-Restauradores}

De este segundo Plan nos interesa recordar que ya recogía como en numerosos foros internacionales se estaba destacado que el verdadero reto de los próximos años residía en la disponibilidad de profesionales que respondieran a una demanda cada vez más diversificada y que implicaba a campos del conocimiento y de la gestión hasta ahora inéditos en la política de los bienes culturales. En este caso se une a las recomendaciones que desde la Unión Europea se hacen en este tema y que ampliaremos un poco más adelante.

También se hace eco de la necesidad de analizar la demanda laboral, la definición de los perfiles profesionales tanto para afianzar las actividades ya consolidadas como para adaptarse a las nuevas expectativas y determinar la enseñanza más apropiada a cada caso según los niveles de formación previa, criterios que deberían estar aplicándose en el campo de la conservación y restauración.

Se evalúan algunos de los problemas que se han presentado en el período del anterior Plan General de Bienes Culturales 89-95 y que al margen de las limitaciones económicas, se pueden sintetizar en los siguientes puntos con relación a la formación y profesionalización de restauradores (pag. 46 y sig.): en cuanto a su planificación y gestión, destaca la ausencia de profesionales de la restauración mueble en la estructura administrativa de las Delegaciones Provinciales de la Consejería de Cultura. Así propone medidas como acomodar la estructura orgánica y mejorar los recursos humanos y los equipamientos de los Servicios Centrales, Delegaciones Provinciales 
e Instituciones del Patrimonio de la Consejería de Cultura y y como proyecto fundamental e imprescindible, elaborar el Reglamento de Conservación y Restauración que determinaría, entre otras cosas, los contenidos exigibles a los proyectos de conservación, los criterios y las normas que aplicarían, etc.

Además se repasa el Programa de Recursos Humanos de la Consejería de Cultura (pág. I 54) y se asume que "en la última Relación de Puestos de Trabajo la Consejería de Cultura tan sólo reconoce 20 plazas de Conservadores-Restauradores para toda Andalucía, sin especificar en la mayoría de los casos los requisito de titulación de las mismas, con estas plazas, algunas sin dotación presupuestaria, se tiene que atender a unas 50 unidades de trabajo con necesidad de estos profesionales". Por último, también se recoge que "es necesario definir los perfiles de los puestos de trabajo, las titulaciones exigibles y la experiencia requerida para desarrollar las actividades en el campo del patrimonio histórico en general y dentro de la Consejería de Cultura en particular, desde la mayor profesionalidad, en concreto en el terreno de la conservación/restauración y en el de la museología" (pág. 155).

No estaría de más recordar los objetivos (pág. I55) que la propia Administración se marcaba, al constatar un déficit de recursos importante en la Consejería de Cultura y la dificultad real de acometer una Plan de estas características a partir de las disponibilidades en ese momento. Entre esos objetivos la Consejería de Cultura se proponía los siguientes:

- Proveerse de personal de plantilla, con dotación de plazas con personal funcionario o laboral, especializado o no, para actividades, técnicas, administrativas, de mantenimiento, etc., suficiente y adecuado a los objetivos, funciones y servicios que tiene que prestar en materia de patrimonio histórico, de acuerdo con las competencias que por ley tiene asignadas y que mantenga la calidad de los servicios, identificando qué funciones mínimas deben ser cubiertas por plantilla para los diferentes servicios e instituciones y que funciones pueden ser descentralizadas, delegadas o contratadas mediante gestión delegada. Pero si repasamos lo que ha ocurrido a día de hoy en la Relación de Puestos de Trabajo (RPT) de la Consejería de Cultura del año en curso "l no hemos encontrado más que seis puestos de trabajo (salvo error en el cómputo), que se adscriben al grupo $\|$ ○ $\mathrm{A}$, en los que con la denominación de restaurador, asesor técnico restauración, título superior o jefe taller-restauración, se cuente con estos profesionales, especificándose sólo en algunos casos como titulación exigida, la de Licenciado en Bellas Artes con formación en restauración. Nos consta que hay alguna plaza más ocupada ya por funcionarios y otras pocas ocupadas con contratos específicos (pero que se renuevan año tras año). ¿Qué ha ocurrido?, pues que oficialmente se ha pasado de esas escasas 20 plazas a casi la mitad en una regresión incomprensible en la que la reorganización de la estructura administrativa interna de la Consejería no ha reforzado la parte técnica y especializada ni la dotación de la relación de puestos de trabajo como se proponía.

- Reforzar la estructura técnica facultativa de las Delegaciones Provinciales de la Consejería de Cultura, lo que hasta el momento se está haciendo con contrataciones específicas de restauradores para realizar labores que requieren una atención continuada y permanente (control y seguimiento de proyectos, propuestas de intervención, etc.).

- Dotar a los profesionales de la Consejería de Cultura de criterios de actuación, conocimientos técnicos y herramientas básicas ajustadas a la realidad de su labor cotidiana, para un mejor desempeño de las tareas que tienen encomendadas en cada centro de trabajo, labor que eficazmente se realiza desde el IAPH, contando con la colaboración de otras instituciones como los Colegios Profesionales, asociaciones profesionales, etc.

- En cuanto a los recursos humanos de la Consejería de Cultura, ésta se proponía la normalización de la contratación de la gestión delegada de servicios en materia de cultura, definiendo los perfiles de los puestos de trabajo, las titulaciones exigibles y la experiencia requerida para desarrollar las actividades en el campo del patrimonio histórico en general y dentro de la Consejería de Cultura en particular (pág. 3|3), labor que sigue pendiente y que como veremos en el caso de la adjudicación de proyectos de intervención mediante concurso, se soluciona con fórmulas de compromiso en las que no queda claro por ejemplo si la titulación requerida es de grado medio o superior.

En la edición digital de la que se han recogido los datos de este segundo Plan, se adjunta además como anexo, la resolución del Parlamento de Andalucía que acordó, como consecuencia del debate del Plan General de Bienes Culturales 1995-2000 12, que durante la vigencia del mismo, el Consejo de Gobierno adoptaría las medidas precisas para consolidar el Instituto Andaluz de Patrimonio Histórico, dotándolo de las infraestructuras y equipamientos necesarios y de los recursos humanos de carácter estable imprescindibles para el desarrollo continuado de sus funciones. Sin embargo parece ser que hasta el momento entre estos recursos no se encuentra, entre otras, la plantilla de restauradores con la que cuenta esta institución.

\section{Los Conservadores-Restauradores y la Administración Pública}

Y ¿cómo se resuelve esta situación en la Administración? El doble nivel formativo existente en España en la actualidad, como Licenciados en Bellas Artes especialidad en Conservación-Restauración y como diplomados en Conservación-Restauración por las Escuelas Superiores, ha propiciado que dos titulaciones con grados diferentes tengan las mismas competencias profesionales cuando acceden a cargos o puestos en la 
Administración Pública. Este acceso se ha solucionada de manera diversa en nuestro país. La Administración Central siempre que contrata restauradores mediante oferta pública de empleo 13 , lo hace adscribiéndolos al grupo II y categoría B. Algunas Administraciones como la del País Vasco o la de Castellón, entre otras, han optado por crear plazas de conservadores-restauradores a dos niveles: A para Licenciados y B para Diplomados. También en Andalucía se ha mantenido este esquema ya que recientemente se ha creado el Cuerpo de Técnicos Superiores en Conservación-Restauración, nivel A para Licenciados, y el Cuerpo de Ayudantes con nivel $B$, aunque actualmente es sólo una declaración de intenciones puesto que no se ha creado ninguna plaza, no hay oposición convocada y el personal que trabaja como técnico lo hace generalmente como laboral dentro del grupo B aun siendo titulados superiores o con contratos específicos. En otras comunidades como Madrid, se han creado puestos, normalmente también de carácter laboral, a los que pueden acceder ambos profesionales, diplomados y licenciados; para éstos, en algún caso como en el MNCARS, se ha intentado una doble adscripción: "asimilados A-B".

En cuanto a la adjudicación de proyectos específicos de intervención de bienes muebles, el problema se resuelve con estas cláusulas-tipo que encontramos en los concursos por procedimiento abierto del Ministerio de Educación y Cultura: en el caso de empresas "será exigible, en cumplimiento de lo dispuesto en el artículo 198.I de la Ley de Contratos con la Administración Pública, que su actividad o finalidad guarde relación directa con el objeto del contrato" o tengan la clasificación que las órdenes de 30 de enero de 1991 y 19 de enero de 1993 exigen; en el caso de los profesionales que "por razón de su titularidad académica de enseñanza universitaria, estén facultadas para la realización del contrato y se encuentren inscritas en el Correspondiente Colegio Profesional, no se les exigirá clasificación". En el caso de adjudicación de expedientes de conservación y restauración de bienes muebles por parte de la Consejería de Cultura de la Junta de Andalucía se recoge que "el adjudicatario del presente trabajo deberá ser una empresa cuyo fin social sea la restauración de bienes muebles o una persona física que esté en posesión del título de restaurador oficialmente reconocido, que representará al equipo de trabajo. Los restauradores que formen el equipo deberán estar igualmente en posesión del título de restaurador oficialmente reconocido".

En ambos casos, este requisito fundamental será de difícil cumplimiento por cuanto han desaparecido esas enseñanzas que otorgan un título oficialmente reconocido, al menos con el grado de licenciatura.

\section{Incorporación de profesionales de la Unión Europea}

Respecto a la incorporación laboral de profesionales de otros países y si ampliamos el marco comparativo a la Unión Europea, vemos que la situación profesional se complica 14. Hoy en día, es impensable la concepción tradicional del restaurador como artesano-profesional que ejecuta su trabajo de forma aislada. Esta concepción tradicional comienza a modificarse en la década de los 80 a través de documentos como el presentado por el Grupo de Trabajo para la formación en Conservación y Restauración del ICOM15 y de la Guía Profesional desarrollada por ECCO 16 y que son los textos básicos de los que se parte conjuntamente con la ya clásica Carta de Venecia de 1964, en las Jornadas tituladas: "Preservación del Patrimonio Cultural: Hacia un perfil europeo del conservador-restaurador", celebradas en Pavia, del 18 al 22 de octubre de 1997. El documento generado en estas Jornadas es a su vez un documento abierto a nuevas líneas de trabajo en la Unión Europea y que se manifiestan a través del Proyecto FULCO de la asociación ENCORE ${ }^{17}$, cuyas líneas principales se sintetizan en un nuevo texto: el Documento de Viena.

En la Unión Europea las desigualdades existentes entre los niveles formativos y el reconocimiento y las competencias en los distintos países están ocasionando una toma de conciencia del problema existente por parte de las distintas Administraciones responsables. En nuestro país se ha detectado además un problema como es el de la dificultad de contratación de ciudadanos europeos al no estar homologados por el Ministerio las titulaciones obtenidas en otros países, ya que lo que se homologa son las asignaturas comunes impartidas en los programas formativos; esto repercute negativamente en la libre circulación de profesionales entre países de la Unión Europea lo que afecta directamente al marco del mercado único.

En el encuentro de Viena, de diciembre de 1998, que promovió el proyecto europeo FULCO y al que acudieron representantes de todos los países del Unión Europea y de las Asociaciones ECCO, ENCORE y la Fundación GIOVIANNI SECCO SUARDO, quedó de manifiesto nuevamente la necesidad del reconocimiento legal de la profesión de conservador-restaurador a nivel europeo, así como la armonización de la formación de conservadores-restauradores a nivel universitario o equivalente reconocido. También hace un llamamiento para que se evite la proliferación de programas formativos sin la adecuada cualificación.

\section{La profesión de Restaurador y su formación oficial}

Como se recoge en el informe-dictamen "Las Enseñanzas Artísticas a examen. Evolución histórica, panorama actual y perspectivas" desarrollado por la Conferencia Sectorial de Educación en julio de 1999, (pág. 35-36), si la conservación y restauración "necesita de la participación de documentalistas, químicos, físicos, petrólogos o biólogos, para establecer la diagnosis previa a cualquier intervención, el eje del diálogo interdisciplinar entre ellos ha de ejercerlo el conservador-restaurador que ha de ensamblar los datos precedentes de los diversos campos, definir las pro- 
puestas globales de tratamiento y, por último, intervenir con la responsabilidad que supone tratar las obras maestras del arte español". Se exige así que "sean técnicos de alto nivel, con dominio de la aplicación de las áreas científicas que les competen y que les permiten diseñar proyectos complejos y distintos". De esta forma se intentaba evitar la paradoja de que siendo los responsables directos de la intervención, estuvieran subordinados a otros especialistas, facilitándose además su acceso a la investigación a través de los estudios de tercer ciclo (doctorado), factor fundamental de desarrollo y progreso de la profesión.

\section{Conclusiones}

Resumiendo, en la Administración Pública, al menos en la autonómica, hasta el momento no se reconoce al conservador-restaurador como cuerpo profesional y si lo hace es contratando a profesionales de la Conservación-Restauración en los dos niveles antes mencionados, licenciado o diplomado sin distinción, variando la categoría administrativa y la asignación económica del puesto que desempeñan, pero no las competencias profesionales ya que ambos están legalmente capacitados para ejercer la profesión, dirigir y ejecutar intervenciones.

Por tanto, desde nuestro Colegio Profesional se está trabajando para que las autoridades competentes promuevan una titulación única con un nivel académico y un contenido formativo común e idóneo a las características del ejercicio de esta profesión y que creemos que debería articularse en dos niveles, titulados superiores y medios (licenciados y diplomados) y que contemple las especialidades necesarias para cubrir la intervención en la totalidad de tipologías de bienes culturales. La Administración tampoco debe olvidarse de articular los mecanismos necesarios para convalidar las titulaciones precedentes impartidas $y / 0$ expedidas por Centros Oficialmente reconocidas, tanto a nivel nacional como, al menos, de la Unión Europea.
También sería competencia de la Administración el instrumentar los mecanismos necesarios para permitir el acceso de estos profesionales al cuerpo de funcionarios públicos mediante la articulación de una vía de acceso acorde con el desarrollo del ejercicio específico de esta profesión, quizá mediante concurso-oposición teórico-práctico. Debe además facilitar los mecanismos de contratación de estos profesionales en los que se valore también su capacitación profesional cuando sea oportuno. También lo es el facilitar el libre acceso de profesionales de otros países de la Unión Europea en igualdad de condiciones que los profesionales españoles y sin que esto afecte negativamente o pueda suponer agravios comparativos al desarrollo profesional de los titulados en España.

Es antigua y reconocida desde la Consejería de Cultura de la Junta de Andalucía, la necesidad de la creación del Reglamento de Conservación y Restauración, objetivo que como ya hemos recogido fue incorporado en el último plan de Bienes Culturales a través de la pregunta parlamentaria de nuestro Colegio Oficial de Doctores y Licenciados en Bellas Artes de Andalucía, con la promesa de nuestra Consejera de una pronta solución y que recordemos nuevamente, debe completar la Ley |//99| de Patrimonio Histórico de Andalucía como instrumento normativo básico, hasta la fecha no se ha formulado ni tan siquiera un borrador.

También ,finalmente para validar un trabajo de los profesionales en la conservación y restauración de Patrimonio ,que se está realizando desde la inversión publica hemos demandado la incorporación de los trabajos con presupuesto del Consejería de Cultura en Anuarios publicados regularmente por esta así como unas jornadas de exposición de proyectos, estudios o intervenciones al igual que vienen celebrando otras disciplinas (arqueología, etnología) no vayamos a caer además de la orfandad formativa en una orfandad informativa que redundaría sin duda en la mejora y formación de esta actividad.

\section{Notas}

I. Lo que se recoge en el artículo 44.I.

2. Documento coordinado por el Instituto Andaluz del Patrimonio Histórico y presentado en las jornadas celebradas en Úbeda y Baeza los días 15 y 16 de febrero de 1996.

3. Plan General de Bienes Culturales 1989-1995, pág. 19.

4. En sesión celebrada los días 12 y 13 de mayo de 1999.

5. Formación que también se imparte en la actualidad desde la Escuelas de Galicia (Pontevedra), Cataluña (Barcelona) y recientemente Baleares.

6. Estudios promovidos por la Ley de Reforma Universitaria (LRU), que se publicaron en el BOE 2370 de 20/I I//990.

7. A través del Real Decreto |387//99| de 18 de septiembre.

8. A través del Decreto 59/1986 de 19 de marzo por el que la Consejería de Cultura aprueba los Estatutos del Patronato

9. Cuadros I y 2 de la pág. 120.

10. Al comienzo del punto 5.3 sobre el Plan de Bienes Muebles (pág. 75).
I1. Publicada en el BOJA 40 de 5 de abril de 2001 .

12. Andalucía 2000, 5-98/PPCG-06383, en sesión celebrada los días 12 y 13 de mayo de 1999.

13. Según información del Jefe de Servicio de Personal Laboral.

14. Tal y como se recoge en el informe del Instituto Andaluz del Patrimonio Histórico: "Titulaciones académicas sobre restauración del patrimonio: problemática y situación actual", marzo de 200 I, Rainiero Baglioni y Mª José González López.

15. Documento titulado: "El conservador-restaurador: Una definición de la profesión" Copenhagen, 1984 y que se consolida en 1993/94, en la "Guía Profesional que elabora la Confederación Europea de Organizaciones de Conservadores-Restauradores (ECCO). En esta Guía se definen las competencias de la profesión, se establece un código deontológico y se relacionan los requisitos básicos para la educación en la Conservación-Restauración.

16. European Confederation of Conservator- Restorers'Organizations.

17. European Network for Conservation-Restauration Education 